\title{
Extended Grasping Behavior for Autonomous Human Agents
}

\author{
Ramon Mas ${ }^{1}$, Ronan Boulic ${ }^{2}$, Daniel Thalmann ${ }^{2}$ \\ ${ }^{1}$ Department of Mathematics and Computer Science, Balearic Islands University, Spain (ramon@anim.uib.es) \\ 2 Computer Graphics Laboratory., Swiss Federal Institute of Technology Switzerland (boulic@lig.di.epfl.ch)
}

\begin{abstract}
In this paper we present an efficient technique allowing distant grasp behaviors while preserving the static balance. This feature greatly increases the autonomy of virtual humans.
\end{abstract}

\section{Introduction}

Our goal is to provide an integrated reaching and grasping behaviors for a virtual human agent. In most cases, grasping a close object only involves the motion of one or both arms often complemented with the independent motion of the head to look towards the goal [RG91][MT94]. However the reach of distant objects is a much more complex task as human beings can extend their field of action by various means. Let us imagine the process of grasping a relatively low object (Fig. 1a). To avoid a loss of balance the body posture is constrained so as to keep the vertical projection of the center of mass inside the base of support (Fig. 1b). However the object can be even more distant and additional supporting area has to be used.(Fig. 1c). In the case of Fig. 1c we can reach the target with the left hand while leaning on the box with the right hand (Fig. 1d). The present approach solves this class of problems on a human body model with 95 degrees of freedom including the hands mobilbities [MT94].

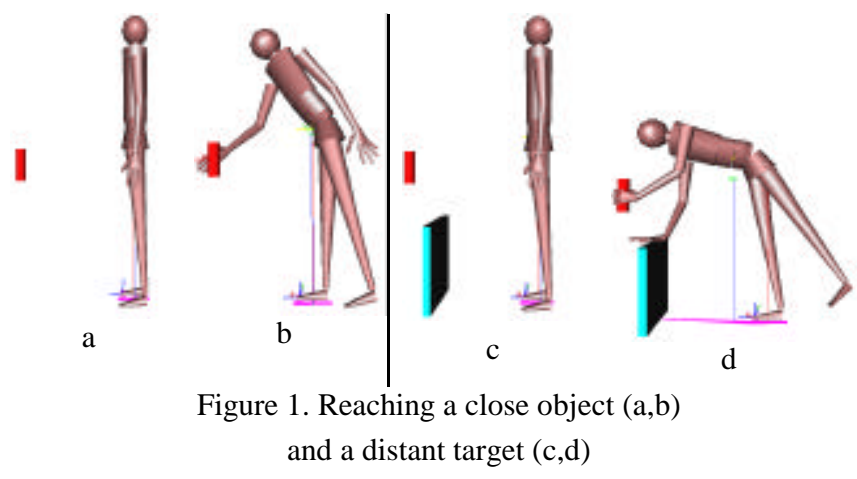

\section{The Grasp Decision Mechanism}

Our approach decomposes into two phases. In the first phase a grasp is selected and the associated hand position defines the desired hand goal. In the second phase the hand goal is used as an additional constraint for our Inverse Kinetics process [BMT95]. We consider the use of basic primitives first proposed by [TBK87] as being the most adequate to help in the grasp choice. Each object has a set of associated volumic primitives where each primitive characterizes a part of the object that can be grasped.
We have adapted a grasp taxonomy [CH89] which provides a classification depending on the degree of precision of the task and on the geometrical characteristics of the objects (Fig. 2).

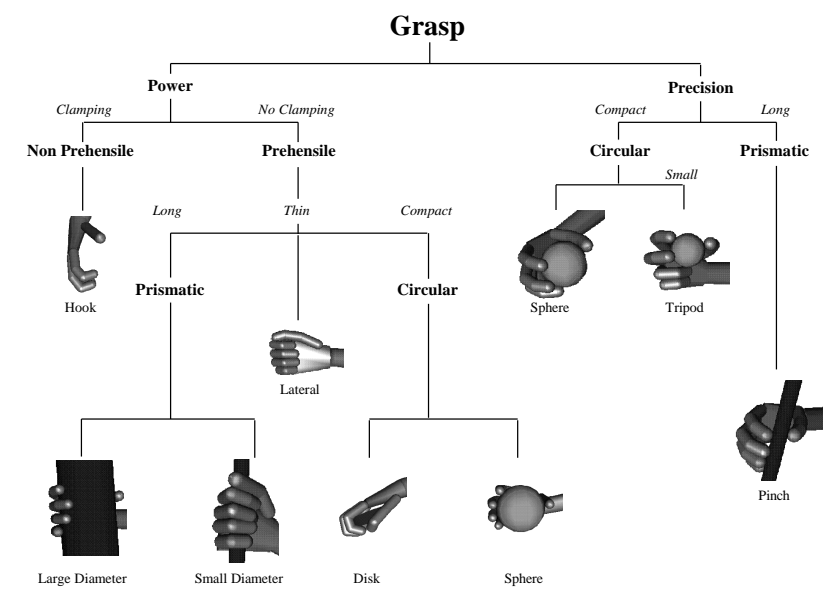

Figure $2:$ The grasp taxonomy

\section{The Reaching Strategy}

The reaching procedure has to bring the hand in a position allowing the grasping of the object. The main factor influencing this procedure is the location of the object relative to the body. Three reach areas are considered : 1) the arm reach area, 2) the Default Body Reach Area with support as in Fig. 1a (in short DBRA), 3) the Extended Body Reach Area with additional support (in short EBRA).

To determine whether the object belongs to the arm reach area is made with a simple test : the distance from the hand to the target is compared to the length of the arm (from the shoulder to the hand). Testing whether the object belongs to the DBRA is difficult to evaluate without a priori knowledge. Moreover, we also have to consider the additional constraint imposed by the balance control requirement and the existence of joint limits. For this reason, we pre-compute the DBRA while standing with balance on both feet

If the object does not belong to the DBRA, new support combinations have to be examined. The starting point is the set of all the possible combinations of the four basic effectors (feet and hands). One of them is always considered as the root for the propagation of the motion, so it has to remain fixed. For this task we give priority to lower limbs, as they are stronger than the upper limbs to sustain the body. Furthermore, the hand used for the grasp cannot be used as a supporting site. So we have only two end effecors to test for selecting the additional support. Distance heuristics are used to create an ordered list of pairs (supporting site, effector) with decreasing probability of fulfilling 
the goal. The best candidate finally determines the motion control structure as illustrated in section 5 .

\section{Inverse Kinetics}

We have proposed a new control technique which takes into account the mass distribution of the body to control precisely the position of the Center of Mass; for this reason we call it Inverse Kinetics [BMT95]. We can guarantee the static balance of the body during the reach process by ensuring that the position of the center of mass lies inside the sustentation polygon. When the body is supported by more than one site, the multiple supports case, we face two problems : 1) How to ensure the geometric constraints of additional support locations ? 2) How to ensure the continuity of the center of mass position control relatively to modifications of the support distribution?

The first problem is a standard Inverse Kinematics problem where the additional supports are considered as end effectors. The second problem requires a center of mass position control depending on the support contribution of each site. Our solution is described in [BMT95].

The reaching behavior emerges from the combined performance of the hand attraction to the target object, with Inverse Kinematics, while the center of mass is retained inside the polygon of sustentation, with Inverse Kinetics. The combination even allow an additional optimization to take place as described in [BMT95].

\section{Simulation Results}

Figure 3 shows grasp postures for different positions of the target object located inside the reachable workspace. In all the cases the body balance is ensured by projecting in the sustentation polygon.



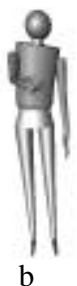

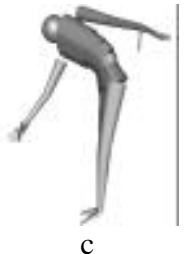

Figure 3 : various reach behaviors of

close objects ensuring the balance
Figure 4 illustrates the animation for grasping a small sphere out of the reachable workspace. First, the grasp decision process selects a tripod as the more convenient mode using Fig. 2 taxonomy. Second, the left hand is activated as the one fulfilling the smallest distance requirement. As the distance from the hand to the target is larger than the arm length, the full body has to be involved. Third, the DBRA voxel data structure is checked and the object is classified outside that space. Fourth, a search on the list of plausible sites within the workspace returns the upper side of the box. Given its location and height it is more suitable for the auxiliary hand than for the leg. Fifth, the additional support center point is computed and assigned as a target to the the right hand. The left leg is allowed to move freely. So the initial state of the motion control is : 1) the left hand has to grasp the sphere using a tripod mode. 2) the right hand has to be placed on the additional support. 3) The balance has the highest priority.

From the default position the human agent moves both hands towards their respective goal while the center of mass is constrained to project in the base of support defined by the right foot (see the vertical line in Fig. 4a and 4b). When the right hand reaches its target (figure 4c) the base of support is enlarged (now it is defined by the projection on the ground of the line joining the right leg and the right hand). Figures $4 b, 4 c$ and $4 d$ show clearly a continuous displacement of the center of mass on that line until the object is reached (use the right foot as a visual reference). When the left hand is at a short distance from the sphere the hand starts closing till the collisions of the fingers with the objects are detected.

The simulation of the sequence takes about 15 seconds on a R4000 computer which is to be compared with the average 6 seconds for a real person to do the same task.

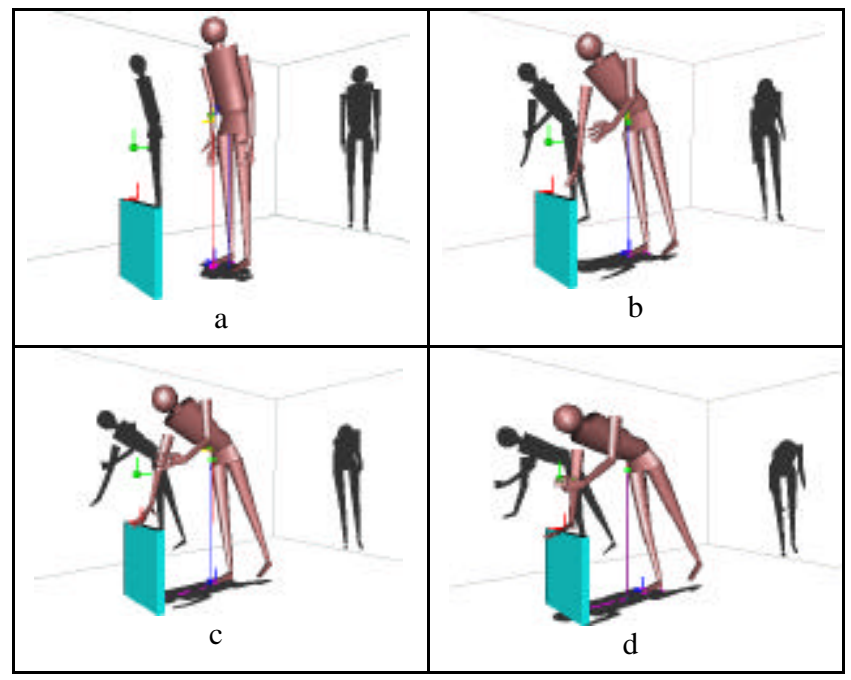

Figure 4: Grasping a distant sphere

\section{Conclusion}

We have presented an integrated reaching and grasping behavior for a virtual human agent. We plan to complete this model with the information of the weight and volume of the grasped object which are very significant to determine the succes of a grasp task.

\section{Acknowledgment}

The research was partly supported by the Swiss National Foundation for Scientific Research.

\section{References}

[BMT95] Boulic, R., Mas, R., Thalmann, D. (1995) "Position Control of the Center of Mass for Articulated Figures in Multiple Support", Eurographics Workshop on Computer Animation and Simulation 1995, D. Terzopoulos \& D. Thalmann Eds., pp 130-143, ISBN 3-211-82738-2, Springer Verlag Wien

[CH89] Cutkosky, M. R., Howe, R. D. (1989). Human Grasp Choice and Robotic Grasp Analysis. In S. T. Venkataraman, \& T. Iberall (Ed.), in "Dextrous Robot Hands" Springer-Verlag.

[MT94] Mas, R., Thalmann, D. (1994). A Hand Control and Automatic Grasping System. Eurographics'94, Computer Graphics Forum, 13(3), pp 167-177.

[RG91] Rijpkema, H., Girard, M. (1991). Computer Animation of Knowledge-Based Human Grasping. Computer Graphics, Las Vegas: ACM SIGGRAPH, pp 339-348.

[TBK87] Tomovic, R., Bekey, G. A., Karplus, W. J. (1987). A Strategy for Grasp Synthesis with Multifingered Robot Hands. Proceedings IEEE on Robotics and Automation, pp 83-89. 